\title{
Reaching the Limit of the Oculomotor Plant: 3D Kinematics after Abducens Nerve Stimulation during the Torsional Vestibulo-Ocular Reflex
}

\author{
Eliana M. Klier, Hui Meng, and Dora E. Angelaki \\ Department of Neuroscience, Baylor College of Medicine, Houston, Texas 77030
}

Accumulating evidence shows that the oculomotor plant is capable of implementing aspects of three-dimensional kinematics such as Listing's law and the half-angle rule. But these studies have only examined the eye under static conditions or with movements that normally obey these rules (e.g., saccades and pursuit). Here we test the capability of the oculomotor plant to rearrange itself as necessary for non-half-angle behavior. Three monkeys (Macaca mulatta) fixated five vertically displaced targets along the midsagittal plane while sitting on a motion platform that rotated sinusoidally about the naso-occipital axis. This activated the torsional, rotational vestibuloocular reflex, which exhibits a zero-angle or negative-angle rule (depending on the visual stimulus). On random sinusoidal cycles, we stimulated the abducens nerve and observed the resultant eye movements. If the plant has rearranged itself to implement this non-halfangle behavior, then stimulation should reveal this behavior. On the other hand, if the plant is only capable of half-angle behavior, then stimulation should reveal a half-angle rule. We find the latter to be true and therefore additional neural signals are likely necessary to implement non-half-angle behavior.

\section{Introduction}

Studies of three-dimensional (3D) eye movements have revealed a remarkable ability of the oculomotor plant to implement 3D kinematics, like the half-angle rule, in the absence of neuronal inputs (Fig. $1 A$, kinematics; Fig. $1 B$, models). First, fibromuscular tissues in the orbits act as pulleys to alter eye muscle pulling direction (Demer et al., 1995), and these pulleys may be actively controlled by the eye muscle's orbital layer (Demer et al., 2000). Second, via single-unit recordings, it has been shown that oculomotor neurons only carry signals related to two-dimensional (2D) eye velocity and not 3D angular eye velocity during pursuit eye movements, as would be necessary to implement Listing's law (Ghasia and Angelaki, 2005). Third, stimulation of the abducens nerve, the final connection between the brain and the oculomotor plant, produces eye movements that obey the half-angle rule both when subjects are upright (Klier et al., 2006) and when they are statically tilted in roll (Klier et al., 2011).

One drawback of the physiological studies above is that they solely examined eye movements that obey Listing's law and the half-angle rule (Fig. 1A), namely saccades and pursuit. An important test would be to stimulate the abducens nerve during eye

Received May 30, 2012; revised July 19, 2012; accepted Aug. 2, 2012.

Author contributions: D.E.A. designed research;H.M. performed research; E.M.K. analyzed data; E.M.K. wrote the paper.

The study was supported by National Institutes of Health Grant R03-010450 to E.M.K. and Grant R01-EY15271 to D.E.A. The authors thank Mykola Kovalenko for his assistance in data collection.

Correspondence should be addressed to Eliana M. Klier, Department of Neuroscience, Baylor College of Medicine, One Baylor Plaza, Houston, TX 77030. E-mail: eklier@cns.bcm.edu.

DOI:10.1523/JNEUROSCI.2595-12.2012

Copyright $\odot 2012$ the authors $\quad 0270-6474 / 12 / 3213237-07 \$ 15.00 / 0$ movements that do not adhere to Listing's law or the half-angle rule and evaluate the resultant stimulation-induced movements. If the plant is truly flexible enough to realign itself as needed, then non-half-angle behavior should be observed. On the other hand, if the plant is designed by default to always implement the halfangle rule, then half-angle behavior will still be realized regardless of the eye movement type.

Eye movements that do not obey Listing's law and the halfangle rule include those movements whose goal it is to keep the eyes on target by compensating for movements of the subject. For example, eye movements induced by either the yaw or pitch rotational vestibulo-ocular reflex (rVOR), exhibit a quarter-angle or zero-angle rule whereby angular eye velocity axes tilt out of Listing's plane, in the same direction as gaze, by only $\leq 25 \%$ of the gaze angle (Misslisch et al., 1994; Thurtell et al., 1999). More dramatically, eye movements induced by the roll rVOR have even more complicated 3D kinematics. Here, depending on whether a visual background is present or not, the eyes either follow a zeroangle rule (where the axis of eye rotation is completely independent of eye position) (Crawford and Vilis, 1991; Misslisch and Hess, 2000) or a negative-angle rule (where the eye's axis moves in the opposite direction to gaze by $25-50 \%$ ) (Misslisch et al., 1994; Misslisch and Hess, 2000). Thus, we chose to stimulate during the roll rVOR since it predicts the largest deviations from the half-angle rule.

Our stimulation results indicate that the oculomotor plant consistently outputs half-angle behavior, even when the task does not warrant it. This implies that this default strategy must be overwritten, likely by neural commands, during non-half-angle behavior. This result has important implications for the mechanical and neural control of 3D motor kinematics. 


\section{Materials and Methods}

Animals and setup. Three male rhesus monkeys (Macaca mulatta) were prepared for 3D eye movement recordings as described by Klier et al. (2006), and these procedures conformed to the guidelines provided by Washington University School of Medicine and the National Institutes of Health.

The monkeys sat such that their heads were in the center of three mutually orthogonal magnetic fields alternating at 60, 90, and 135 $\mathrm{Hz}$ (CNC Engineering), and the signals generated by the magnetic fields in the search coils were detected by three-axis phase-sensitive detectors (CNC Engineering). The magnetic field frame was mounted on an Acutronic rotary motion platform, capable of sinusoidally rotating the animal in roll (i.e., rotation about the naso-occipital axis). A flat-screen computer monitor was also mounted on the motion platform, outside of the magnetic field frame, on which targets were presented.

Experimental protocol. The abducens nerve was chosen for several reasons. First, for the halfangle rule to be examined, angular eye velocity must be evaluated at different vertical eye positions. Thus, it was necessary to elicit eye movements that do not change initial vertical fixation. The abducens nerve, which innervates the lateral rectus muscle is ideal since it abducts the eye, producing only horizontal eye movements. On a related note, the lateral rectus muscle does not produce changes in torsional eye position, which is what needs to be measured to compute the half-angle rule. Also, unlike the oculomotor nerve, the abducens nerve controls a single muscle, so its stimulation produces a simple, stereotyped eye movement. Finally, the abducens nerve is readily accessible, identifiable, and isolated enough so that current does not spread to other nerves.

The abducens nucleus was identified by its characteristic burst-tonic activity during ipsilateral eye movements. Subsequently, the abducens nerve was found by searching for similar activity $\sim 2 \mathrm{~mm}$ anterior, lateral, and ventral to the abducens nucleus. The right abducens nerve was stimulated in monkey $\mathrm{F}$ and the left abducens nerve was stimulated in monkeys D and O. Once identified, the following biphasic stimulation parameters were applied using a microelectrode: pulse duration $=0.2$ $\mathrm{ms}$, train duration $=50 \mathrm{~ms}$, frequency $=500$ $\mathrm{Hz}$. Note that we chose these short-duration, high-frequency parameters to generate highvelocity movements with small displacements. High-velocity eye movements are desirable since the half-angle rule is evaluated by examining the tilts of angular eye velocity. But since changes in angular eye velocity are referenced to initial eye position, it is also important to avoid large changes in eye position to keep the analysis accurate. The stimulation current, which never exceeded $75 \mu \mathrm{A}$, was determined by finding threshold current level and subsequently stimulating at 1.5 times threshold.

The monkeys were required to fixate a central visual target presented on a flat-screen monitor at a distance of $29 \mathrm{~cm}$ in front of them. Along with the onset of the central target, the chair began to rotate sinusoidally in roll, at a frequency of $1 \mathrm{~Hz}$, to an amplitude of $\pm 5^{\circ}$ (Fig. $2 A$ ). The monkeys had to maintain fixation of the central target during the chair

\section{Side view}

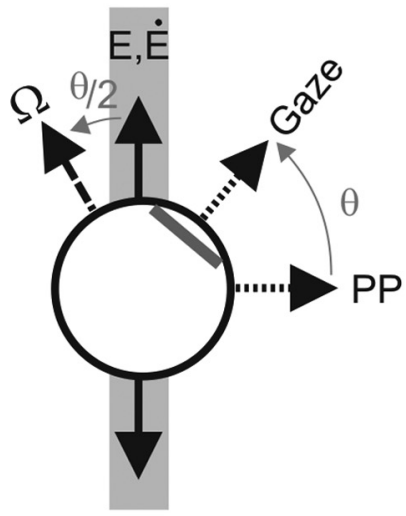

Now

Figure 1. 3D kinematics and their implementation. $\boldsymbol{A}$, Front view, Movements such as saccades and pursuit obey Listing's law, which states that the eye only rotates about axes that lie in a common plane (gray surface), known as Listing's plane (eye positions on this surface have zero torsional components). These axes of rotation follow the right-hand rule: place the right thumb along one of the straight arrows and the fingers curl in the direction of motion that takes the eye from primary position (PP) to its new position (circular arrows). The length of the arrow indicates the amplitude of rotation. Side view: In order for these eye positions (E) to remain in Listing's plane, angular eye velocities $(\Omega)$ must tilt out of Listing's plane by half the amount of gaze eccentricity from PP (i.e., the half-angle rule). This is because the angular velocity of rotational objects is mathematically noncommutative and thus depends on both the derivative of eye position ( $\dot{E}$, which does lie in Listing's plane) as well as initial eye position (E). B, These kinematics were originally believed to be completely neurally driven (for both visually guided and VOR movements), with the correct 3D, noncommutative commands input to an oculomotor plant with eye position-independent muscle pulling directions (Original). Subsequently, several studies both supported and contradicted a revised scheme with a commutative brain for visually guided eye movements and an adjustable oculomotor plant capable of being both position-dependent for incoming 2D visually guided eye movements and position-independent for incoming 3D vestibular commands (Newer). Our results support the newest scheme, proposed by Demer (2006), in which the various neural inputs converge onto an oculomotor plant that is obligated to implement the half-angle strategy via eye position-dependent eye muscle pulling directions (Now).

motion, and fixation was determined automatically by monitoring the location of the monkeys' eyes relative to a theoretical window of $3^{\circ} \times 3^{\circ}$. Randomly, every three to six cycles, stimulation was delivered at one of four different time markers: positive peak position (PPP), negative peak position (NPP), positive peak velocity (PPV), and negative peak velocity (NPV) (Fig. 2A, example of a PPP stimulation trial). The different time markers were used to stimulate at different torsional eye positions and velocities (Fig. $2 \mathrm{~B}$ ). Indeed, average torsional eye position was $-2.46^{\circ} \pm$ $0.37^{\circ}$ during PPP stimulation versus $1.11^{\circ} \pm 1.37^{\circ}$ during NPP stimulation, and these values were significantly different $(p=0.03)$. Average torsional eye velocity was $-20.07^{\circ} \pm 4.29^{\circ} / \mathrm{s}$ during PPV stimulation versus $17.62^{\circ} \pm 2.68^{\circ}$ s during NPV stimulation, and these values were 
A Example of PPP Stimulation

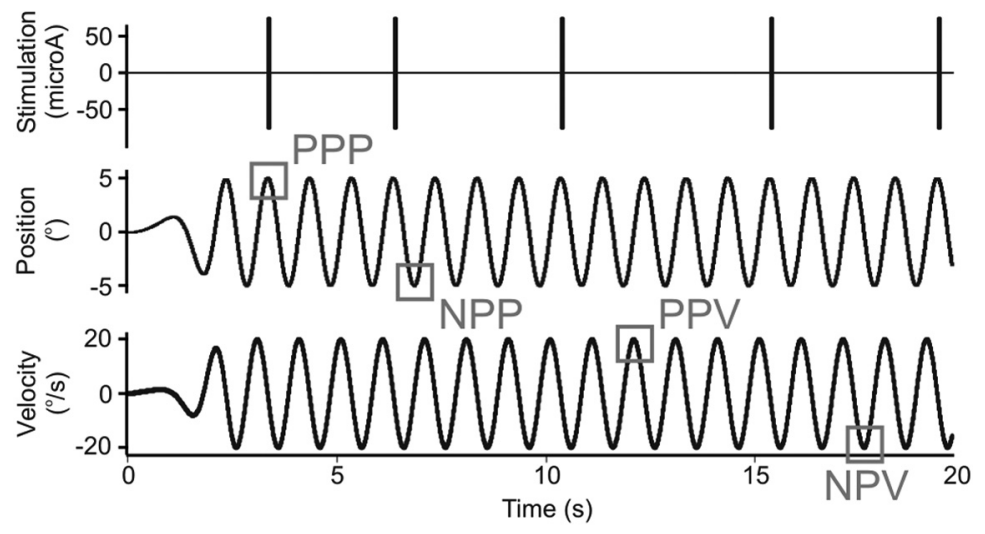

B
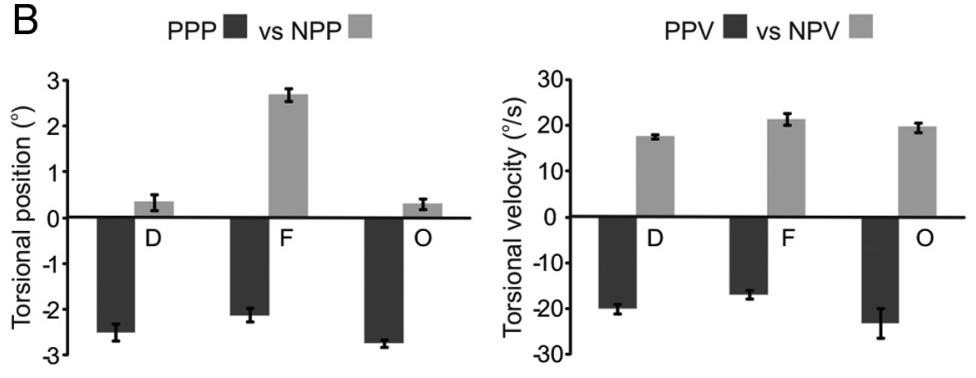

Figure 2. A, Experimental design. The motion platform rolled sinusoidally at a frequency of $1 \mathrm{~Hz}$, to a maximum amplitude of $\pm 5^{\circ}$. Chair position (middle plot) and chair velocity (bottom plot) are illustrated for a PPP trial. Stimulations (top plot) were delivered randomly every three to six trials, resulting in both stimulation and nonstimulation data within in each trial. The onset of stimulation coincided with one of four time markers (gray boxes): PPP (illustrated here), NPP, PPV, and NPV. B, Eye positions and velocities at stimulation onset. Average torsional eye position is shown for all three monkeys (D, F, and 0 ) at PPP and NPP stimulation onset (left). Average torsional eye velocity is shown at PPV and NPV (right). Positive values indicate clockwise direction and negative values indicate counterclockwise direction. Error bars reflect $\pm \mathrm{SE}$.

also significantly different $(p<0.001)$. A motivational juice reward was given whenever the monkey's eye remained in the window continuously for $2 \mathrm{~s}$ (equivalent to two sinusoidal cycles). Stimulations were repeated 5-10 times for each of the four different time markers. Thus, for each time marker, there were many more nonstimulation cycles than stimulation cycles (Fig. 2A). Stimulus presentation, juice delivery, and data acquisition were controlled with custom-written scripts in Spike2 (Cambridge Electronics Design [CED]) using a CED Power 1401. Data were antialias filtered $(200 \mathrm{~Hz}$, six-pole Bessel filter) and then digitized by the CED program at a rate of $833.33 \mathrm{~Hz}$ (16-bit resolution) and recorded on a computer for off-line analysis.

At the beginning of each experimental day, a calibration was performed in which the animals were required to fixate 13 computergenerated targets along the horizontal and vertical meridians $\left(0^{\circ}, \pm 10^{\circ}\right.$, $\left.\pm 15^{\circ}, \pm 20^{\circ}\right)$. At the end of each experiment, in an upright orientation, spontaneous eye movements (i.e., without microstimulation) were also recorded to measure Listing's plane and primary position (Klier et al., 2006). This was done by having the monkeys fixate targets in a $5 \times 5$ array as they were turned on randomly, one at a time, on the monitor, and spanned a visual range of $\pm 20^{\circ}$ horizontally and vertically.

Finally, on two nonexperimental days, nonstimulation saccade data were collected from monkeys $\mathrm{F}$ and $\mathrm{O}$. Here, monkeys made $5^{\circ}$ saccades (from center to $5^{\circ}$ left) at five different vertical eccentricities $\left(0^{\circ}\right.$, $\left.\pm 10^{\circ}, \pm 20^{\circ}\right)$. Half-angle rule measurements of these data were used as control comparisons for the stimulation data. This was not done for monkey D.

Data analysis. Raw eye coil signals were first calibrated (Klier et al., $2005)$ and converted into rotation vectors. These rotation vectors were then rotated into Listing's coordinates and angular eye velocity was computed. The formulas for these procedures are outlined in Klier et al. (2011).
The dependence of eye velocity on eye position during the VOR could not be immediately computed since the monkeys were engaged in a roll rVOR task when the stimulation was delivered. Thus, the recorded eye movements were comprised of two separate components. First, the roll rVOR caused the eye to rotate torsionally, about an axis equal and opposite to that of head/body rotation, and second, stimulation of the abducens nerve caused a mainly horizontal change in eye position. Thus, it was first necessary to compute an average, nonstimulated trajectory from the nonstimulated trials and subsequently subtract it from each individual stimulation-induced trajectory, thus ensuring that the remaining movement is comprised only of a stimulationinduced movement. The subtraction was done component by component in the torsional, vertical, and horizontal dimensions (e.g., the averaged, nonstimulated torsional component was subtracted from each individual stimulationinduced torsional component). This subtraction is critical because the experiment hinges on examining the outcome of the stimulation-induced movement alone, which provides a snapshot of the organization of the oculomotor plant at the time of stimulation.

Once these stimulation-induced movements were filtered, eye velocity dependence could be determined by plotting horizontal versus torsional eye velocity. These traces were drawn from stimulation onset to peak velocity (i.e., showing just the stimulation-induced movement). For simplicity, we decided not to show the subsequent passive eye movement that returns the eye to a more central position in the orbits, but we have previously shown that measures of the half-angle rule with and without this passive section are equivalent (Klier et al., 2006). Subsequently, linear fits (type II regression) were made to these data and the tilt angle of these regression lines from Listing's plane was measured. Finally, these tilt angles were plotted as a function of vertical eye position, and a second linear fit (type I regression) was made to these data, the slope of which served as the measure of eye position dependence (e.g., a slope of 0.5 indicates the half-angle rule) (Klier et al., 2011).

\section{Results}

The main goal of the experiment is to determine whether the eyes' axes of rotation, during movements that do not obey Listing's law and the half-angle rule, are determined by ocular mechanics or other, likely neural, sources. This question can be answered by examining how electrical stimulation-induced angular velocity, which defines the axis of eye rotation, changes as a function of vertical eye position. But first, the stimulation-induced eye movement had to be isolated from the recorded eye movement which also contained an additional torsional component because the monkey was engaged in a roll rVOR task.

Once eye position and angular velocity were computed in Listing's coordinates, plots of torsional, vertical, and horizontal position and velocity components were made as a function of time for both stimulation and nonstimulation trials. Figure 3 shows these traces for a one-time marker (PPV) in monkey D. Here, stimulation data are aligned on the stimulation onset that coincides with PPV (Fig. $3 A, B$, left columns), while nonstimulation trials are also aligned on PPV (but no stimulation was delivered) (Fig. $3 A, B$, right columns). Notice that the position traces (Fig. $3 A$ ) indicate very little move- 


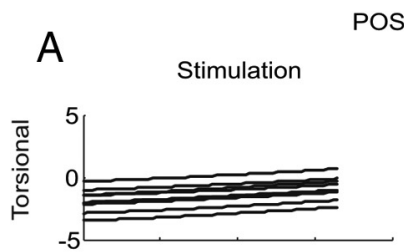

POSITION ( $\left.{ }^{\circ}\right)$
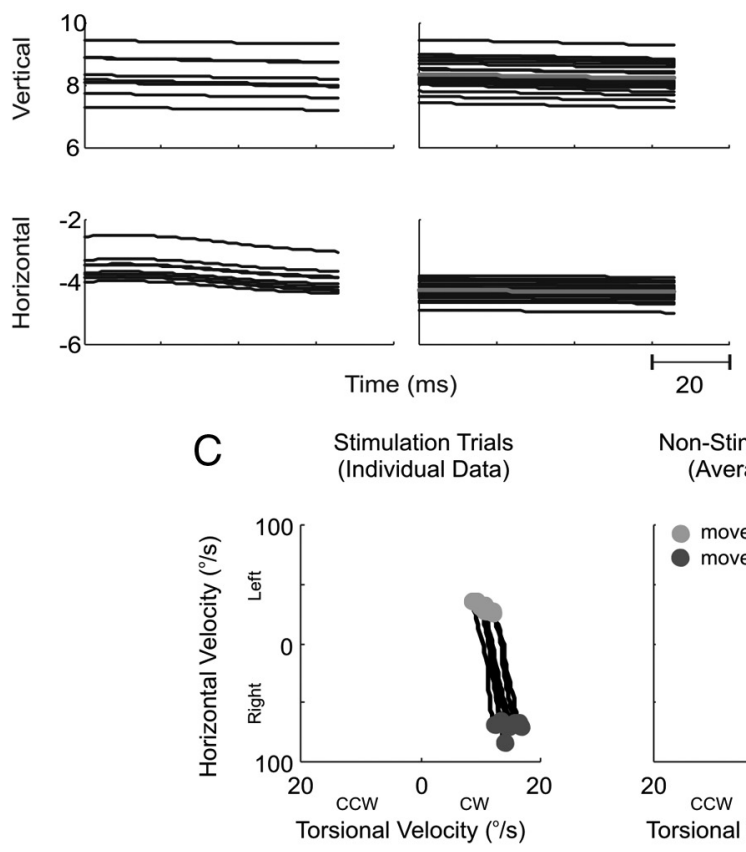

Non-stimulation

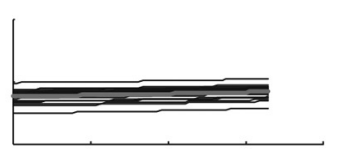

Non-Stimulation Trials (Averaged Data)

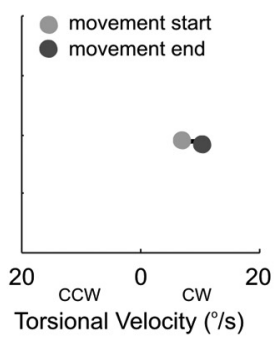

VELOCITY $(\%)$

B
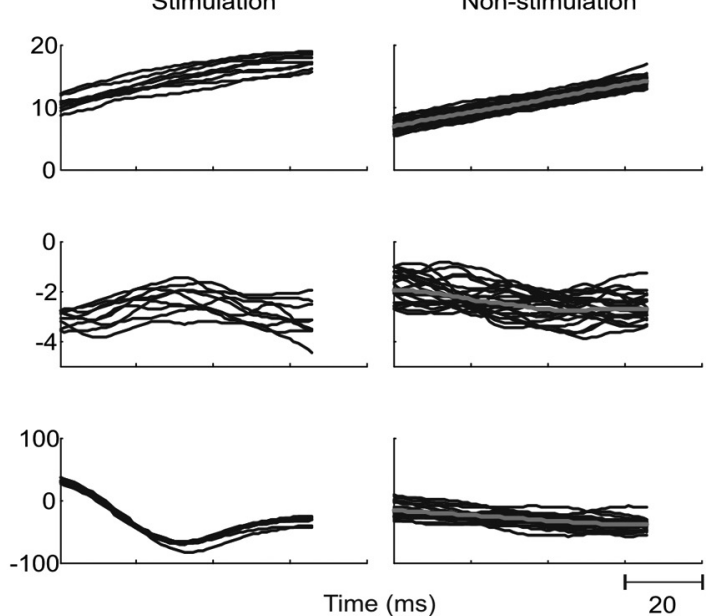

Non-stimulation

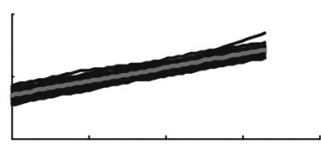

Stimulation Trials Minus Non-Stimulation Average

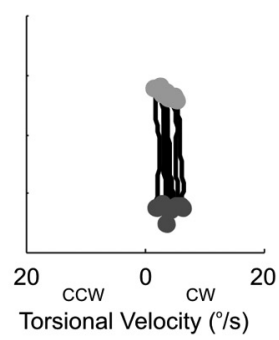

Figure 3. Isolating the stimulation-induced eye movements. $\boldsymbol{A}, \boldsymbol{B}$, Temporal plots of position $(\boldsymbol{A})$ and velocity $(\boldsymbol{B})$ are illustrated for a sample trial in monkey $D$. Torsional, vertical, and horizontal components of both stimulation (left columns) and nonstimulation (right columns) data are shown. Stimulation traces are plotted from the onset of stimulation, which is coincident with one of the four time markers, while nonstimulation traces are plotted from corresponding time markers. Gray lines indicate the average of nonstimulation trials. $C$, Spatial plots of horizontal versus torsional traces of eye velocity are shown for stimulation trials (left), averaged nonstimulation trials (middle), and subtracted results (right). The start of each movement is indicated by the light gray circles, and the end of the movements by the dark gray circles.

ment, whereas the horizontal velocity traces show the most displacement in the horizontal component during stimulation trials (Fig. 3B, left column), as would be expected with our stimulation parameters in the abducens nerve. Next, the nonstimulation position and velocity trajectories were averaged (Fig. $3 A, B$, right columns, gray traces superimposed on torsional, vertical, and horizontal components) to provide a typical example of the eye's behavior during the roll rVOR alone.

The aforementioned temporal, stimulation-induced, velocity traces were converted into spatial plots of horizontal versus torsional eye velocity (Fig. 3C, left column). Similar plots of averaged, nonstimulation-induced movements were also plotted (Fig. 3C, middle column). These began at the same time marker as stimulation movements (i.e., PPP, NPP, PPV, or NPV) and were duration matched to the corresponding stimulation movement. Then, these averaged nonstimulation data were subtracted from individual stimulation trials, leaving only the stimulation-induced component of the eye movement (Fig. 3C, right column).

The left column of Figure 4 plots horizontal versus torsional eye velocity from the onset of the time marker coincident with stimulation onset [PPV (first row), NPV (second row), PPP (third row), or NPP (fourth row)] to the peak velocity of the stimulation-induced movement, for one experimental day in monkey $\mathrm{O}$. The different colors indicate different vertical gaze eccentricities [i.e., where the monkey was looking when the stimulation was delivered; dark blue $=20^{\circ}$ up; light blue $=10^{\circ}$ up; green $=0^{\circ}$ (i.e., straight ahead); pink $=10^{\circ}$ down; red $=20^{\circ}$ down], and their tilting pattern shows a dependency of the axes of rotation on these vertical gaze positions.

Regression lines were fit to each stimulation-induced eye velocity axis, and the tilt angle of these regression lines from upright were then plotted as a function of eye position [Fig. 4, right column (same conventions as in the left column where different colors indicate different vertical gaze eccentricities)]. Regression lines were then fit to the resultant data (solid black lines), and the slope of these regression lines indicate the degree to which the velocity axes depend on eye position. Across all conditions, the average slope for monkey $\mathrm{O}$ was $0.76 \pm 0.04$, a value that is not significantly different from the control eye position slope of $0.72 \pm 0.12$ computed for this animal's naturally generated, saccadic eye movements $(p=0.19)$. Average eye position slopes for animal F were $0.62 \pm 0.06$ (stimulation-induced) and $0.58 \pm 0.18$ (control), which were also not significantly different $(p=0.27)$. The average, stimulation-induced slope for monkey D was $0.37 \pm 0.07$. Data for all monkeys, in all conditions, are shown in Table 1 . Notice that each monkey showed its own distinct half-angle behavior that was consistent across the four different time markers, as well as similar to the stimulationinduced eye velocity slope with the animal upright and stationary [Table 1 (data from Klier et al., 2011)]. As explained previously (Klier et al., 2006), this reflects the distinctiveness of each monkey's oculomotor plant (some of which may have been slightly 

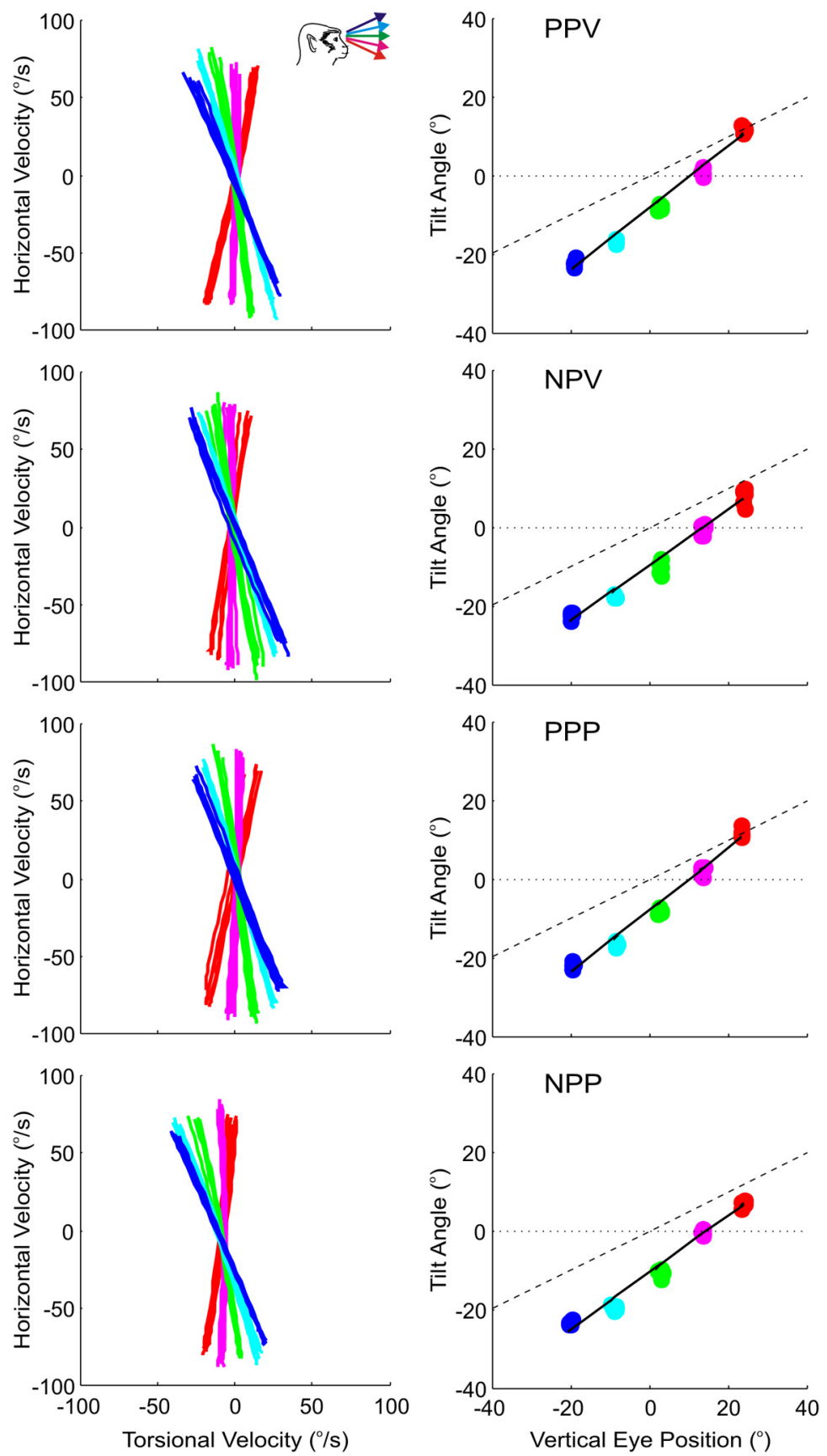

Figure 4. Computing the eye position dependence of stimulation-induced eye movements. Left column, Horizontal versus torsional plots of eye position are plotted and color coded according to vertical eye position (see monkey inset; dark blue $=20^{\circ}$ up; light blue $=10^{\circ}$ up; green $=0^{\circ} ;$ pink $=10^{\circ}$ down; red $=20^{\circ}$ down). Right column, The tilt angles of the traces in the left column are plotted as a function of vertical eye position. A regression line is fit to these data (solid black line), and, for comparison, a slope of 0.5 is indicated by the dashed black line. Each row indicates data from a different time marker (PPV, NPV, PPP, and NPP). Data are from monkey 0 .

altered during their eye coil surgeries). The average stimulation slope across all three animals was $0.58 \pm 0.16$.

\section{Discussion}

Our evidence supports the idea that the oculomotor plant is uniquely designed to implement eye velocity axes that adhere to the half-angle rule. This is the case even when the active eye movement itself does not obey the half-angle rule, as is the case with the torsional rVOR. This default oculomotor strategy may be helpful as it may simplify the work of the brain for some eye movements, namely fixations, saccades, and pursuit (Quaia and Optican, 1998). But for others, like the rVOR, neural drive is likely necessary to direct appropriately in $3 \mathrm{D}$, despite a default half-angle rule configuration of the eye plant.

\section{The role of the oculomotor pulleys}

Since the first mention of oculomotor pulleys (Miller, 1989), great progress has been made to understand the contribution of the oculomotor plant to $3 \mathrm{D}$ eye movement kinematics. Static pulleys have given way to the active pulley hypothesis, which explains how the pulleys of the recti muscles can implement the half-angle rule at all possible eye positions (Demer et al., 2000; Kono et al., 2002). For eye movements that normally obey Listing's law, the four rectus muscles appear to operate as an inner gimbal, enforcing the halfangle rule. Our previous neurphysiological studies are in agreement with this view (Ghasia and Angelaki, 2006; Klier et al., 2006, 2011). However, the role of the pulleys in implementing non-listing behavior is not as clear. It has been hypothesized that the oblique eye muscles act as an outer gimbal, rotating the inner gimbal as necessary to implement static ocular counter-roll and the quarter-angle (or zero-angle) rule of the rVOR (for review, see Demer, 2006), although experimental evidence has been available only for the former (Demer and Clark, 2005; Klier et al., 2011).

Eye movement kinematics have been studied dynamically during the yaw rVOR (Crane et al., 2005). Here, eye positions driven out of Listing's plane remained out of, but on a parallel surface to, Listing's plane during subsequent saccades. This could only occur if the eye continued to follow the half-angle rule on the displaced surface. This finding is in complete agreement with our current results that the plant is uniquely designed to always follow the half-angle rule.

Ultimately, the movement of the pulleys must be observed dynamically, during non-half-angle behavior, but this is not possible naturally via magnetic resonance imaging since the head must remain stable. In addition, it is unlikely that new anatomical structures will be found to explain quarter-angle or zero-angle behavior. Focus must now shift to the underlying neural activity and its differential control of the orbital and global layers that innervate the pulleys and eye muscles, respectively (Porter et al., 1995; Demer et al., 2000).

\section{Neural signals for the rVOR}

Several studies have examined neural discharge during ocular counter-roll. They have found that superior colliculus cells 
Table 1. Measures of the half-angle rule during the roll rVOR and saccades

\begin{tabular}{|c|c|c|c|c|c|c|}
\hline \multirow[b]{2}{*}{ Animal } & \multicolumn{4}{|l|}{ Torsional rVOR } & \multirow[b]{2}{*}{ Saccades (control) } & \multirow{2}{*}{$\begin{array}{l}\text { Fixated + stimulatec } \\
\text { (Klier et al., 2011) }\end{array}$} \\
\hline & PPV & NPV & PPP & NPP & & \\
\hline D & $0.37 \pm 0.20$ & $0.42 \pm 0.11$ & $0.40 \pm 0.24$ & $0.37 \pm 0.07$ & DNC & $0.59 \pm 0.06$ \\
\hline $\mathrm{F}$ & $0.67 \pm 0.05$ & $0.58 \pm 0.04$ & $0.66 \pm 0.04$ & $0.56 \pm 0.03$ & $0.58 \pm 0.18$ & $0.65 \pm 0.09$ \\
\hline 0 & $0.79 \pm 0.04$ & $0.71 \pm 0.05$ & $0.79 \pm 0.04$ & $0.73 \pm 0.04$ & $0.72 \pm 0.12$ & $0.77 \pm 0.06$ \\
\hline
\end{tabular}

Mean values ( $\pm 95 \%$ confidence intervals) of the half-angle rule for the four different time markers (PPV, NPV, PPP, and NPP), shown separately for each animal (D, F, and 0$)$. Control data for monkey D was not collected (DNC, did not collect). Data are also shown from Klier et al. (2011), where the abducens nerve was stimulated while the monkey, in an upright orientation, fixated targets along the midline at five vertical eccentricities $\left(0^{\circ}, \pm 10^{\circ}\right.$, and $\pm 20^{\circ}$.

(Frens et al., 1998) and burst neurons (Scherberger et al., 2001) shift their preferred directions during static head tilt. In addition, eye-head neurons, a premotor cell type that is the target of Purkinje cell inhibition from the cerebellar flocculus/ ventral paraflocculus, exhibit properties that could be consistent with the half-angle rule during smooth pursuit eye movements (Ghasia et al., 2008). It was thus suggested that eye-head cells may be functionally related to the output of a forward internal model thought to construct an efference copy of the actual eye movement. In contrast, another group of pre-motor neurons called burst-tonic cells, like extraocular motoneurons (Ghasia and Angelaki, 2005), do not carry signals appropriate for the half-angle rule of ocular kinematics during smooth-pursuit eye movements from eccentric positions. It remains to be seen whether cyclovertical motoneurons carry the proper torsional commands to elicit appropriate 3D kinematics during the rVOR. For example, it is possible that the horizontal VOR, unlike horizontal saccades and pursuit, also activates oblique motoneurons such that the half-angle rule is altered using appropriate 3D neural commands. Future recordings from extraocular motoneurons during the VOR should provide valuable information in this regard.

Thus, while it may be true that realigning the rectus gimbal via pulley shifts during the rVOR would maintain apparent plant commutativity (Quaia and Optican, 1998), it is clear that the brain must grapple with torsional control issues. For example, the rVOR must still generate 3D commands to the eyes that are equal and opposite to that of head rotation, and head-free gaze shifts somehow produce predictive torsional movements out of Listing's plane in the first part of a gaze shift that are later nullified by VOR-related eye movements in the second part of the gaze shift (Crawford et al., 1999). In addition, combinations of rVOR (which does not follow the half-angle rule) and translational VOR (which follows the half-angle rule; Angelaki et al., 2003) clearly point to the need of precisely controlled neural signals (Angelaki, 2003).

\section{Extrapolation to other effectors}

The fact that peripheral anatomy strongly influences 3D oculomotor behavior makes it compelling to search for mechanical and neural correlates of torsional control in other motor systems. For example, the head (Radau et al., 1994) and arm (Hore et al., 1992) follow a more relaxed form of Listing's law known as Donder's law (where torsion at each unique orientation is always constant, but it need not be zero). However, Donder's law is broken when rolling the head (e.g., the right ear touches the right shoulder) or when the outstretched arm is rotated about the index finger. Both mechanical and neural contributions to the $3 \mathrm{D}$ constraints of these systems have not yet been studied.

Finally, on a related note, these findings pose interesting evolutionary questions since it appears that the control of phylogenetically newer eye movements like saccades and pursuit is relegated to the periphery, whereas older eye move- ments like the VOR are centrally controlled. As with all evolutionary questions, it is important not to mix up cause and effect. For example, it has been speculated that the head obeys Donders' law because of the Fick strategy imposed on head movements through the anatomical positioning of the first two cervical vertebrae-the axis and the atlas (Richmond and Vidal, 1998). Thus, it may not be that an evolutionary force caused the half-angle rule to be relegated to the oculomotor plant, but rather that the pre-existing anatomical arrangement of the rectus muscles (i.e., the inner gimbal) lent itself to the emergence of Listing's law and the half-angle rule.

\section{References}

Angelaki DE (2003) Three-dimensional ocular kinematics during eccentric rotations: evidence for functional rather than mechanical constraints. J Neurophysiol 89:2685-2696.

Angelaki DE, Zhou HH, Wei M (2003) Foveal versus full-field visual stabilization strategies for translational and rotational head movements. J Neurosci 23:1104-1108.

Crane BT, Tian J, Demer JL (2005) Kinematics of vertical saccades during the yaw vestibulo-ocular reflex in humans. Invest Ophthalmol Vis Sci 46:2800-2809.

Crawford JD, Vilis T (1991) Axes of eye rotation and Listing's law during rotations of the head. J Neurophysiol 65:407-423.

Crawford JD, Ceylan MZ, Klier EM, Guitton D (1999) Three-dimensional eye-head coordination during gaze saccades in the primate. J Neurophysiol 81:1760-1782.

Demer JL (2006) Current concepts of mechanical and neural factors in ocular motility. Curr Opin Neurol 19:4-13.

Demer JL, Clark RA (2005) Magnetic resonance imaging of human extraocular muscles during static ocular counter-rolling. J Neurophysiol 94:3292-3302.

Demer JL, Miller JM, Poukens V, Vinters HV, Glasgow BJ (1995) Evidence for fibromuscular pulleys of the recti extraocular muscles. Invest Ophthalmol Vis Sci 36:1125-1136.

Demer JL, Oh SY, Poukens V (2000) Evidence for active control of rectus extraocular muscle pulleys. Invest Ophthalmol Vis Sci 41:1280-1290.

Frens MA, Suzuki Y, Scherberger H, Hepp K, Henn V (1998) The collicular code of saccade direction depends on the roll orientation of the head relative to gravity. Exp Brain Res 120:283-290.

Ghasia FF, Angelaki DE (2005) Do motoneurons encode the noncommutativity of ocular rotations? Neuron 47:281-293

Ghasia FF, Meng H, Angelaki DE (2008) Neural correlates of forward and inverse models for eye movements: evidence from three-dimensional kinematics. J Neurosci 28:5082-5087.

Hore J, Watts S, Vilis T (1992) Constraints on arm position when pointing in three dimensions: Donders' law and the Fick gimbal strategy. J Neurophysiol 68:374-383.

Klier EM, Angelaki DE, Hess BJ (2005) Roles of gravitational cues and efference copy signals in the rotational updating of memory saccades. J Neurophysiol 94:468-478.

Klier EM, Meng H, Angelaki DE (2006) Three-dimensional kinematics at the level of the oculomotor plant. J Neurosci 26:2732-2737.

Klier EM, Meng H, Angelaki DE (2011) Revealing the kinematics of the oculomotor plant with tertiary eye positions and ocular counterroll. J Neurophysiol 105:640-649. 
Kono R, Clark RA, Demer JL (2002) Active pulleys: magnetic resonance imaging of rectus muscle paths in tertiary gazes. Invest Ophthalmol Vis Sci 43:2179-2188.

Miller JM (1989) Functional anatomy of normal human rectus muscles. Vision Res 29:223-240.

Misslisch H, Hess BJ (2000) Three-dimensional vestibuloocular reflex of the monkey: optimal retinal image stabilization versus Listing's law. J Neurophysiol 83:3264-3276.

Misslisch H, Tweed D, Fetter M, Sievering D, Koenig E (1994) Rotational kinematics of the human vestibuloocular reflex. III. Listing's law. J Neurophysiol 72:2490-2520.

Porter JD, Baker RS, Ragusa RJ, Brueckner JK (1995) Extraocular muscles: basic and clinical aspects of structure and function. Surv Ophthalmol 39:451-484.
Quaia C, Optican LM (1998) Commutative saccadic generator is sufficient to control a 3-D ocular plant with pulleys. J Neurophysiol 79:3197-3215.

Radau P, Tweed D, Vilis T (1994) Three-dimensional eye, head, and chest orientations after large gaze shifts and the underlying neural strategies. J Neurophysiol 72:2840-2852.

Richmond FJR, Vidal PP (1998) The motor system: joints and muscles of the neck. In: Control of head movement (Peterson BW, Richmond FJR, eds), pp 1-21. New York: Oxford UP.

Scherberger H, Cabungcal JH, Hepp K, Suzuki Y, Straumann D, Henn V (2001) Ocular dounterroll modulates the preferred direction of saccaderelated pontine burst neurons in the monkey. J Neurophysiol 86:935-949.

Thurtell MJ, Black RA, Halmagyi GM, Curthoys IS, Aw ST (1999) Vertical eye position-dependence of the human vestibuloocular reflex during passive and active yaw head rotations. J Neurophysiol 81:2415-2428. 